\title{
Control parasitario en perros y gatos: conocimiento sobre las principales enfermedades parasitarias en el sureste mexicano
}

\author{
Parasitic control in dogs and cats: knowledge about the major parasitic diseases in \\ the Mexican Southeast
}

\author{
L.A. Encalada-Mena ${ }^{1,2}$, J.J. Vargas-Magaña ${ }^{1}$, I.E. Duarte-Ubaldo ${ }^{1}$, \\ M.J. García-Ramírez ${ }^{1}$
}

\section{Resumen}

\begin{abstract}
El objetivo del estudio fue determinar los programas de control parasitario, sanitario y conocimiento de las enfermedades parasitarias que afectan a perros y gatos de 306 propietarios de la ciudad de Escárcega, estado de Campeche, México. El total de mascotas fue de 590 perros y 149 gatos. Cada hogar tiene un promedio de 1.92 perros o 0.48 gatos con edades de 2.9 y 2.8 años, respectivamente. La mayoría de los propietarios de perros $(30.8 \%)$ y gatos $(25.6 \%)$ aplican algún tratamiento antihelmíntico dos veces al año. La frecuencia de tratamientos contra ectoparásitos en perros y gatos se realiza mayormente cuatro veces al año o al detectar su presencia. Los parásitos gastrointestinales son poco conocidos y la sarna es la enfermedad más mencionada. La mayoría de los propietarios de perros $(85.6 \%)$ y gatos $(93 \%)$ desconocen el significado de zoonosis y que sus mascotas les pueden transmitir enfermedades, lo que sugiere su bajo impacto sobre el manejo sanitario en las mascotas. Lactonas macrocíclicas como endoparasiticidas y el amitraz y propoxur como ectoparasiticidas fueron los más usados para perros y gatos.
\end{abstract}

Palabras clave: perros; gatos; manejo sanitario; control; enfermedades parasitarias; zoonosis

\section{Abstract}

The aim of this study was to determine the programmes of parasite and sanitary control and knowledge of the parasite diseases that affect dogs and cats of 306 owners of the city of Escárcega, state of Campeche, Mexico. The total number of pets was 590 dogs and 149 cats. Each household has an average of 1.92 dogs or 0.48 cats with ages of

\footnotetext{
${ }^{1}$ Escuela Superior de Ciencias Agropecuarias, Universidad Autónoma de Campeche, Escárcega, Campeche, México

${ }^{2}$ E-mail: laencala@uacam.mx
}

Recibido: 28 de enero de 2019

Aceptado para publicación: 6 de septiembre de 2019 
2.9 and 2.8 years, respectively. Most owners of dogs (30.8\%) and cats (25.6\%) apply some anthelmintic treatment twice a year. The frequency of treatments against ectoparasites in dogs and cats is carried out mostly four times a year or when their presence is detected. Gastrointestinal parasites are poorly known, and scabies is the most mentioned disease. Most owners of dogs (85.6\%) and cats (93\%) are unaware of the meaning of zoonosis and that their pets can transmit diseases to them, suggesting its low impact on health management in pets. Macrocyclic lactones as endo-parasiticides and amitraz and propoxur as ecto-parasiticides were the most used for dogs and cats.

Key words: dogs, cats; health management; control; parasitic diseases; zoonoses

\section{INTRODUCCIÓN}

La convivencia de las mascotas con el hombre ha demostrado indudables beneficios económicos y culturales; sin embargo, estas mascotas se ven afectadas por diferentes agentes parasitarios que afectan su salud y, que si no son controladas pueden ocasionar problemas en la salud, bienestar y la seguridad de sus propietarios, sobre todo en zonas donde su tenencia y reproducción no es controlada (Arauco et al., 2014). A pesar de los enormes esfuerzos del hombre por eliminar a los parásitos de sus mascotas, estos continúan siendo un grave problema de salud en países desarrollados (Matos et al., 2015) y este impacto es mucho más notorio en países en vías de desarrollo (Encalada-Mena et al., 2011).

Los endoparásitos se encuentran asociados a problemas gastroentéricos, al sistema circulatorio, así como a otros tejidos y órganos, en tanto que los ectoparásitos están asociados mayormente a problemas de piel (Bowman, 2011; Matos et al., 2015). Los perros y gatos son especies que han sido asociadas con un gran número de enfermedades parasitarias que impactan a nivel global (Chomel, 2014; Otranto et al., 2017), especialmente las coccidias, Giardia, Ancylostoma sp, Toxocara sp, Dipylidium $\mathrm{sp}$, Ctenocephalides sp y garrapatas (Rodríguez-Vivas et al., 2001; EncaladaMena et al., 2011; Dantas-Torres y Otranto,
2014; Alvarado-Esquivel et al., 2015). La importancia que representan estos parásitos ha llevado a la European Scientific Counsel Companion Animal Parasites (ESCCAP) para los países de la Unión Europea y la Companion Animal Parasite Council (CAPC Vet) para los Estados Unidos y Canadá a crear guías sobre la manera de controlar las parasitosis en las mascotas, además de proporcionar medidas higiénicas sanitarias para disponer de las heces de las mascotas.

Estas instancias recomiendan tratamientos antiparasitarios cada semana hasta los 14 días posdestete en cachorros y al menos cuatro veces al año para animales adultos; sin embargo, es importante considerar que los tratamientos frecuentes pueden llevar al desarrollo de resistencia antihelmíntica (RA) (Kopp et al., 2008; Riggio et al., 2013). En los países en vías de desarrollo la situación se complica, ya que se debe considerar el crecimiento incontrolado de la población canina y felina en situación de calle, y que una gran cantidad de perros de casa tienen acceso al exterior, lo que aumenta la posibilidad de contagio entre animales y al humano (Ortega-Pacheco et al., 2007; Acosta-Jammet et al., 2010).

La mejor manera de lograr programas preventivos de éxito es a través de la concientización de los propietarios por intermedio de los médicos veterinarios sobre la cantidad de enfermedades parasitarias que pueden afectar a sus mascotas y su poten- 
cial contagio hacia ellos mismos (zoonosis) (Awosanya y Akande, 2015; Diez et al., 2015), así como las medidas higiénico-sanitarias que se deben tomar sobre los desechos, especialmente las heces de las mascotas, como potenciales fuentes de contagio (manejo y eliminación de deshechos en casa y al salir de paseo) (Penakalapati et al., 2017). Este programa de concientización requiere conocer el sustento que usan los propietarios de mascotas para proporcionarles tratamientos preventivos (Belshaw et al., 2018). Sin embargo, en el sureste mexicano no se conoce la forma en que los propietarios de mascotas protegen a sus animales de estos parásitos (diagnóstico, tratamiento y frecuencia), ni la manera en que manejan los desechos de los animales de compañía. Debido a lo anterior, el presente estudio tuvo como objetivo determinar los programas de control parasitario que los propietarios de perros y gatos usan en sus mascotas, su conocimiento sobre las enfermedades parasitarias que pueden afectarlos y las medidas de control sanitario que aplican para evitar contagios.

\section{Materiales y Métodos}

\section{Localización y Hogares}

El estudio se realizó entre noviembre y diciembre de 2016 en el sureste de México, en la ciudad de Escárcega, localizada en el Municipio del mismo nombre, perteneciente al estado de Campeche, México. Cuenta con un clima cálido subhúmedo con lluvias en verano. La temperatura promedio anual es de $26.1^{\circ} \mathrm{C}$ con valores máximos y mínimos de 27.6 y $23.9^{\circ} \mathrm{C}$, respectivamente (Anuario estadístico y geográfico de Campeche, 2017). Cuenta con una población de 29477 habitantes que representan el $54 \%$ de la población total del municipio (SEDESOL, 2013). La ciudad se caracteriza por sus casas de tipo rural ubicadas en medio de amplios solares, en su mayoría de un solo nivel y provistas de vegetación y árboles frutales, característicos del sistema de producción denominado huerto familiar o «solar» (Montañez-Escalante et al., 2014).

\section{Encuesta Piloto}

En virtud de que no existe en Escárcega alguna investigación que señale el número de casas que cuentan con mascota, dato requerido para obtener el tamaño mínimo de muestra, se realizaron encuestas a tres hogares aleatoriamente en cada una de las 20 colonias de la ciudad. Se determinó que las personas entrevistadas fueran los jefes de familia o personas mayores de 18 años. Las preguntas de la encuesta estuvieron dirigidas para saber si tenían perros o gatos como mascotas, determinándose que el $71.5 \%$ del total de encuestados tenían al menos una mascota (perro o gato).

\section{Tamaño de Muestra}

Con base a los resultados de la encuesta piloto, se determinó el tamaño de muestra requerido utilizando la fórmula de muestreo aleatorio simple en poblaciones finitas con los siguientes datos: probabilidad de casas que tiene perros y gatos (71.5\%), tamaño de la población (13 282 casas) (INEGI, 2010), margen de error $(5 \%)$ y nivel de confianza (95\%). Como resultado se encontró la necesidad de encuestar a 306 hogares en las 20 colonias.

\section{Entrevista}

Para la selección de los hogares a encuestar se usaron los mismos criterios que en la encuesta piloto, cuidando encuestar al menos 15 hogares por colonia (a veces fueron 16) para completar el tamaño de muestra. Se utilizó un formato de entrevista semiestructurada con 30 preguntas: 6 dicotómicas, 3 abiertas y 21 de opción múltiple de una sola respuesta desarrolladas por el grupo de trabajo y que se validó a través de la encuesta piloto. La encuesta tomó entre 15 y 20 minutos en completarse. Se tomaron en cuenta varios apartados tales como: 
i) Estatus social: edad, último grado de estudios, género, número de habitantes en el hogar (4 preguntas); ii) Salud de mascotas: conocimiento sobre los parásitos gastrointestinales y ectoparásitos que suelen tener sus mascotas, enfermedades más comunes causadas por estos parásitos, conocimiento general sobre las enfermedades transmisibles de las mascotas a los humanos (zoonosis) (16 preguntas), donde los entrevistados contestaban de manera libre, previa explicación, el concepto de zoonosis; iii) Profilaxis: conocimiento de los fármacos que utilizan para desparasitarlos, frecuencia con que desparasitan a sus mascotas, practicas sanitarias y visitas a los veterinarios para desparasitar las mascotas (10 preguntas).

\section{Análisis Estadístico}

Se obtuvo estadística descriptiva (media, desviación estándar, proporciones e intervalos de confianza del 95\%) para la mayoría de las variables el programa estadístico SPSS $15.0^{\circledR}$. Se elaboraron cuadros de contingencia y se estableció la asociación entre variables mediante la prueba de Chi cuadrado, donde un valor de $\mathrm{p}<0.05$ fue considerado como significativo.

\section{Resultados}

\section{Aspecto Social}

Se entrevistaron a 205 mujeres $(66.9 \%)$ y 101 hombres $(33.1 \%)$ con edad promedio de 37.3 años y mediana de 36 años. El nivel de escolaridad fue el siguiente: sin estudios $19(6.2 \%)$, primaria $75(24.5 \%)$, secundaria 89 (29.1), bachillerato 84 (27.4\%) y licenciatura $39(12.8 \%)$. En cada hogar se determinó que habitan 2.6 adultos y 1.8 menores de edad.
Cuadro 1. Frecuencia de perros y gatos hallados en 306 hogares entrevistados en la ciudad de Escárcega, Campeche, México (2016)

\begin{tabular}{ccccc}
\hline Mascotas & \multicolumn{2}{c}{ Perros } & \multicolumn{2}{c}{ Gatos } \\
\cline { 2 - 5 } & $\mathrm{n}$ & $\%$ & $\mathrm{n}$ & $\%$ \\
\hline 0 & 16 & 5.2 & 205 & 66.9 \\
1 & 107 & 35.0 & 67 & 21.9 \\
2 & 100 & 32.7 & 22 & 7.2 \\
3 & 49 & 16.0 & 10 & 3.3 \\
4 & 34 & 11.1 & 2 & 0.7 \\
\hline Hogares & 306 & 100.0 & 306 & 100.0 \\
\hline
\end{tabular}

Cuadro 2. Frecuencia de tratamiento contra helmintos gastroentéricos en perros y gatos de 306 hogares de la ciudad de Escárcega, Campeche, México (2016)

\begin{tabular}{cccccc}
\hline \multirow{2}{*}{$\begin{array}{c}\text { Tratamientos } \\
\text { por año }\end{array}$} & \multicolumn{2}{c}{ Perros } & \multicolumn{2}{c}{ Gatos } & \multicolumn{2}{c}{ Total } \\
\cline { 2 - 6 } & $\mathrm{n}$ & $\%$ & $\mathrm{n}$ & $\%$ & $\mathrm{n}$ \\
\hline 0 & 52 & 19.8 & 17 & 39.5 & 69 \\
1 & 66 & 25.1 & 8 & 18.6 & 74 \\
2 & 81 & 30.8 & 11 & 25.6 & 92 \\
3 & 40 & 15.2 & 4 & 9.3 & 44 \\
4 & 20 & 7.6 & 2 & 4.7 & 22 \\
$>4$ & 4 & 1.5 & 1 & 2.3 & 5 \\
\hline Hogares & 263 & & 43 & & 306 \\
\hline
\end{tabular}

\section{Mascotas}

Se determinó que los propietarios prefieren tener de uno a dos perros (1.92 perros) y en el caso de los gatos, la mayoría no tiene o prefiere tener solo uno ( 0.48 gatos) (Cuadro 1). La edad promedio de 590 perros y 149 gatos fue 2.9 y 2.8 años, respectivamente. El $61.2 \%$ de los perros fue machos y $38.8 \%$ hembra $(p<0.05)$, en tanto que el 
Cuadro 3. Frecuencia de tratamiento contra ectoparásitos en perros y gatos de 306 hogares de la ciudad de Escárcega, Campeche, México (2016)

\begin{tabular}{cccccc}
\hline \multirow{2}{*}{$\begin{array}{c}\text { Tratamientos } \\
\text { por año }\end{array}$} & \multicolumn{2}{c}{ Perros } & \multicolumn{2}{c}{ Gatos } & Total \\
\cline { 2 - 6 } & $\mathrm{n}$ & $\%$ & $\mathrm{n}$ & $\%$ & $\mathrm{n}$ \\
\hline 0 & 38 & 14.4 & 16 & 37.2 & 54 \\
1 & 4 & 1.5 & 3 & 7.0 & 7 \\
2 & 19 & 7.2 & 1 & 2.3 & 20 \\
3 & 9 & 3.4 & 1 & 2.3 & 10 \\
4 & 57 & 21.7 & 10 & 23.3 & 67 \\
5 & - & - & - & - & - \\
6 & 24 & 9.1 & 3 & 7.0 & 27 \\
$>6$ & 51 & 19.4 & 1 & 2.3 & 52 \\
Ocasional $^{1}$ & 61 & 23.2 & 8 & 18.6 & 69 \\
\hline Hogares & 263 & \multicolumn{5}{c}{43} & 306 \\
\hline${ }^{1}$ Cuando observan presencia de ectoparásitos $^{2}$
\end{tabular}

Cuadro 4. Fármacos contra endo- y ectoparásitos usados por los propietarios de mascotas de la ciudad de Escárcega, Campeche, México (2016)

\begin{tabular}{lcccc}
\hline \multirow{2}{*}{ Fármacos } & \multicolumn{2}{c}{ Perros } & \multicolumn{2}{c}{ Gatos } \\
\cline { 2 - 5 } & $\mathrm{n}$ & $\%$ & $\mathrm{n}$ & $\%$ \\
\hline Endoparasiticidas & & & & \\
$\begin{array}{l}\text { Lactona macrocíclica } \\
\text { sola }\end{array}$ & 11 & 30.6 & 2 & 50.0 \\
Lactona macrocíclica & 4 & 11.1 & 1 & 25.0 \\
combinada & & & & \\
Albendazol & 8 & 22.2 & 1 & 25.0 \\
$\begin{array}{l}\text { Febendazol } \\
\text { Pirantel + febendazol }+\end{array}$ & 1 & 2.8 & 0 & 0 \\
prazicuantel & 5 & 13.9 & 0 & 0 \\
$\begin{array}{l}\text { Prazicuantel + } \\
\text { pirantel }\end{array}$ & 5 & 13.8 & 0 & 0 \\
\hline \multicolumn{1}{c}{ Total de hogares } & 34 & & 4 & \\
\hline Ectoparasitidas & & & & \\
Fipronil & 4 & 14.3 & 2 & 25.0 \\
Amitraz & 9 & 32.1 & 0 & 0 \\
$\begin{array}{l}\text { Propoxur } \\
\text { Coumafos }\end{array}$ & 3 & 10.7 & 3 & 37.5 \\
Lactona macrociclica & 7 & 25.0 & 3 & 37.5 \\
sola & 5 & 17.9 & 0 & 0 \\
\hline \multicolumn{1}{c}{ Total de hogares } & 28 & & 8 & \\
\hline
\end{tabular}

$51.2 \%$ de los gatos fue macho y el $48.8 \%$ fue hembra. Así también, 28\% (87) de los hogares tenían ambas especies, pero para los fines de este estudio los datos se muestras por especie.

\section{Endoparasitosis en las Mascotas}

En el Cuadro 2 se observa que la mayor frecuencia de tratamiento antihelmíntico en las mascotas, tanto en perros como gatos es de dos veces al año en los hogares encuestados. El $80.2 \%$ de los perros es desparasitado entre 1 y más de 4 veces al año, mientras que el $60.5 \%$ de los gatos es desparasitado entre 1 y más de 4 veces al año.

De los 237 hogares que afirman aplicar tratamiento contra helmintos, 135 (56.9\%) 1leva su mascota al veterinario y el resto $(n=102$, $43.1 \%$ ) adquieren el medicamento en alguna agro-veterinaria o farmacia veterinaria y lo administran ellos mismos. De los que llevan su mascota al veterinario, solo 71 (52.5\%) realizan un examen rutinario de las heces. Por otro lado, solo 40 (16.8\%) afirman conocer el nombre comercial o químico del tratamiento aplicado.

\section{Ectoparasitosis en las Mascotas}

El 85.6\% de los hogares con perros y el $62.5 \%$ de aquellos con gatos tratan a sus mascotas contra ectoparásitos. La mayoría realiza estos tratamientos contra ectoparásitos cuatro veces al año o cuando observan su presencia, con ligeras diferencias entre perros y gatos (Cuadro 3).

En el caso de los 252 propietarios que afirman aplicar tratamiento contra ectoparásitos, 38 (15.1\%) acuden al veterinario y 214 (84.9\%) lo adquieren en alguna agro-veterinaria o farmacia veterinaria y lo administran ellos mismos. Solamente 36 (14.3\%) conocen el fármaco que aplican para el control de ectoparásitos. 
Cuadro 5. Manejo de las heces de las mascotas (perros y gatos) en 306 hogares de la ciudad de Escárcega, Campeche, México

\begin{tabular}{llcccc}
\hline \multirow{2}{*}{ Pregunta } & & \multicolumn{2}{c}{ Perros } & \multicolumn{2}{c}{ Gatos } \\
\cline { 2 - 6 } & & $\mathrm{n}$ & $\%$ & $\mathrm{n}$ & $\%$ \\
\hline ¿En dónde defecan? & Patio & 202 & 76.8 & 25 & 58.1 \\
(lugar más común) & Calle y lugares públicos & 61 & 23.2 & 13 & 30.2 \\
& Arenero & 0 & 0 & 5 & 11.6 \\
¿Se deshacen de & $\mathrm{Si}$ & 183 & 69.6 & 26 & 60.5 \\
las heces? & No & 80 & 30.4 & 17 & 39.5 \\
Tratamiento de & Cubren con cal & 18 & 6.8 & 1 & 2.3 \\
las heces & Cubren con tierra & 40 & 15.2 & 4 & 9.3 \\
& Dejan secar y luego & & & & \\
& recogen y tiran a la & 19 & 7.2 & 3 & 7.0 \\
& basura & & & & \\
& Inmediatamente recogen & 106 & 40.3 & 18 & 41.9 \\
\hline Total de hogares & y tiran a la basura & & & 43 & \\
\hline
\end{tabular}

Cuadro 6. Relación entre el manejo sanitario, conocimiento de enfermedades y esterilización de mascotas con el nivel de estudios de 306 dueños de mascotas de la ciudad de Escárcega, Campeche, México (2016)

\begin{tabular}{llccc}
\hline Variable & Nivel de estudio & No & Sí & $p$ \\
\hline Desparasita contra & Sin estudios & 4 & 15 & 0.0002 \\
helmintos & Primaria & 31 & 44 & \\
& Secundaria & 16 & 73 & \\
& Bachillerato & 15 & 69 & \\
& Licenciatura & 3 & 36 & \\
Controla pulgas y & Sin estudios & 7 & 12 & 0.0189 \\
garrapatas & Primaria & 17 & 58 & \\
& Secundaria & 18 & 71 & \\
& Bachillerato & 8 & 76 & \\
Conoce alguna & Licenciatura & 4 & 35 & \\
enfermedad zoonótica & Sin estudios & 13 & 6 & 0.3885 \\
& Primaria & 46 & 29 & \\
& Secundaria & 62 & 27 & \\
& Bachillerato & 58 & 26 & \\
& Licenciatura & 21 & 18 & \\
Esterilizan mascotas & Sin estudios & 15 & 4 & 0.0095 \\
& Primaria & 60 & 15 & \\
& Secundaria & 83 & 6 & \\
& Bachillerato & 80 & 4 & \\
& Licenciatura & 33 & 6 & \\
\hline
\end{tabular}


Cuadro 7. Conocimiento de enfermedades zoonóticas parasitarias en 306 hogares con mascotas (perros y gatos) en la ciudad de Escárcega, Campeche, México (2016)

\begin{tabular}{lcccc}
\hline & \multicolumn{2}{c}{ Perros } & \multicolumn{2}{c}{ Gatos } \\
\cline { 2 - 5 } & $\mathrm{n}$ & $\%$ & $\mathrm{n}$ & $\%$ \\
\hline Sarna & 27 & 10.3 & 2 & 4.7 \\
Parasitosis gastroentéricas & 4 & 1.5 & 0 & 0 \\
Giardiasis & 2 & 0.8 & 0 & 0 \\
Hemoparásitos transmitidos por garrapatas & 3 & 1.1 & 0 & 0 \\
Toxoplasmosis & 2 & 0.8 & 1 & 2.3 \\
Desconocen alguna enfermedad zoonótica parasitaria & 225 & 85.6 & 40 & 93.0 \\
\hline Total de hogares & 263 & & 43 & \\
\hline
\end{tabular}

\section{Fármacos Utilizados}

Los fármacos más utilizados como endoparasiticidas fueron las lactonas macrocíclicas y como ectoparasitidas el amitraz y el propoxur, tanto para perros como para gatos (Cuadro 4).

\section{Manejo de las Heces}

La mayoría de las mascotas defecan en el patio de los hogares, así como en la calle y lugares públicos y solamente el 40 y $41 \%$ de los propietarios de perros y gatos, respectivamente, se deshacen de las heces apropiadamente (Cuadro 5).

\section{Relación del Nivel Educativo con Otras Variables}

El nivel educativo y el uso de la desparasitación de mascotas contra helmintos resultaron estar están asociados $(\mathrm{p}<0.05)$, lo que indica que los propietarios de mascotas con mayor nivel educativo tienen una mayor conciencia sobre el cuidado de las mascotas. La misma situación se presentó en el control de pulgas y garrapatas, aunque en este caso la relación no fue estadísticamente significativa.

Los mayores porcentajes de esterilización de las mascotas se presentaron en los entrevistados sin estudios y nivel primaria. Aun así, el porcentaje de propietarios que recurren a la esterilización de las mascotas fue bajo ( $\mathrm{n}=35,11.4 \%)$. Por otro lado, el conocimiento de enfermedades zoonóticas no tuvo relación con el nivel de estudio del entrevistado (Cuadro 6).

\section{Conocimiento de Enfermedades Zoonóticas Parasitarias}

El $87 \%(n=265)$ de los propietarios de mascotas desconocen sobre las enfermedades parasitarias que transmiten las mascotas. Aun así, la sarna fue la enfermedad más conocida por los propietarios con 29 (9.5\%), seguida de parasitosis gastroentéricas $(1.3 \%)$, hemoparasitosis (1\%) y finalmente giardiasis $\mathrm{y}$ toxoplasmosis (Cuadro 7).

\section{Discusión}

\section{Mascotas}

Los resultados hallados se encuentran cercanos a los rangos y acordes a dos regiones de México donde encontraron 1.78 perros por hogar en Morelos (Orihuela y Solano, 1995) y entre 1.6 y 2.9 perros por hogar en Yucatán (Ortega-Pacheco et al., 2007). Así también, la edad de los perros estuvo dentro del rango de 2.5 y 3.1 encontrado por Ortega-Pacheco et al. (2007) para la zona 
urbana y rural. respectivamente. La relación macho/hembra fue de 1.63:1.0 en los perros, semejante a muchas ciudades de países en vías de desarrollo, donde se observa una mayor presencia de machos respecto de hembras (Orihuela y Solano, 1995; Morales et al., 2009; Ruiz-Izaguirre y Eilers, 2012), dado que los machos son considerados mejores compañeros de trabajo y porque no entran en celo (Becker et al., 2012). La proporción macho/ hembra en gatos fue de 1.04:1.0, lo cual indica que no existen preferencias por determinado sexo (Toribio et al., 2009), y el resultado se encuentra dentro del rango encontrado en el trabajo de revisión de Rinzin (2007).

\section{Endoparasitosis}

Con respecto al uso de medicamentos endoparasiticidas en perros, los resultados encontrados son menores al $86 \%$ reportado en Finlandia (Pullola et al., 2006), 87\% en Suiza (Sager et al., 2005) y $86.5 \%$ en Portugal (Matos et al., 2015) de perros desparasitados 1-2 veces al año. Por el contrario, Palmer et al. (2010) reportaron solo el $54 \%$ de perros desparasitados, pero con una frecuencia de cuatro veces al año. En cuanto a los gatos, Matos et al. (2015) y Pereira et al. (2016), ambos en Portugal, reportan resultados semejantes a los del presente estudio (63.6 y $71.1 \%$ de desparasitación, respectivamente), pero con una frecuencia mensual o cada cuatro meses. Por otro lado, Beugnet et al. (2014) indican que el 65.8\% de los gatos en varios países de Europa son tratados, la mayoría con 1 o 2 tratamientos al año (Beugnet et al., 2014), situación semejante al presente estudio.

Los resultados de la encuesta reflejan el desconocimiento de los propietarios sobre el uso recomendado de los antihelmínticos. Al respecto, Elsheikha y Jiménez (2015) mencionan que, para lograr avances en el control de los parásitos, el clínico no debe considerar la desparasitación como un procedimiento de rutina basado solamente en la examinación de los signos clínicos, sino incluir la revisión exhaustiva de los pacientes abarcando aspec- tos como: estilo de vida, historial de viajes y los tratamientos previos, sin excluir detalles relevantes. Además los veterinarios deben informar a los dueños sobre la importancia de la evaluación coproparasitológica y el tratamiento periódico a las mascotas (www.esccap.org; www.capcvet.org). La encuesta indica que solo el 4.7 y $7.6 \%$ de los entrevistados desparasitan a sus gatos y perros trimestralmente, lo cual es un indicio de la necesidad de un mayor conocimiento por parte de los dueños (Palmer et al., 2010) para poder mejorar los programas de desparasitación de las mascotas.

\section{Ectoparasitosis}

Matos et al. (2015) reportan que el 92.2\% de los entrevistados en Portugal aplican tratamiento contra ectoparásitos en perros y gatos, en tanto que Beugnet et al. (2014) mencionan que todos los gatos (100\%) en su estudio a nivel de Europa son tratados contra ectoparásitos. Así mismo, las frecuencias de desparasitación en perros y gatos reportada por Matos et al. (2015) y de gatos en Portugal por Pereira et al. (2016) mencionan mayores frecuencias de desparasitación que las encontradas en el presente estudio. No se dispone de estudios que estimen la prevalencia de ectoparásitos en los perros y gatos de la región, pero se puede suponer que podría estar entre el 35 y $80 \%$ en mascotas de regiones tropicales (CostaJunior et al., 2012; Prates et al., 2013; Murthy et al., 2017). Por otro lado, se puede afirmar que el $100 \%$ de los perros y gatos en el estudio no son tratados contra ectoparásitos según la frecuencia mensual recomendada por lineamientos internacionales (www.esccap.org, www.capcvet.org), lo que provoca un mayor riesgo de infestación en las mascotas de la región y en los hogares donde conviven seres humanos y animales (Troyo et al., 2012). Además, se debe considerar el riesgo de propagación de enfermedades transmitidas por vectores como bartonelosis y ricketsiosis (Low et al., 2017; Otranto et al., 2017), las cuales también son potencialmente zoonóticas. 
El bajo porcentaje de dueños de mascotas que acuden al veterinario (15.1\%) y el escaso conocimiento del fármaco que aplican (14.3\%) permite suponer que la mayoría de los propietarios podrían estar realizando un manejo no adecuado de los productos y que podrían estar obteniendo una baja efectividad de los fármacos utilizados (Halos et al., 2014).

\section{Fármacos Utilizados en la Despara- sitación}

Resultados diferentes a los reportados en este estudio fueron encontrados por Matos et al. (2015) y Pereira et al. (2016), quienes mencionan las combinaciones de prazicuantel, pirantel y febantel son los más frecuentes en perros y las lactonas macrocíclicas y milbemicina oxima en gatos para tratamientos contra endoparásitos. Así mismo, estos autores reportan el empleo de imidacloprid solo o combinado para ambas especies como el ectoparasiticida más utilizado.

\section{Manejo de las Heces}

El resultado más sobresaliente es el alto porcentaje ( $60 \%$ en ambas especies) de propietarios que no se deshacen adecuadamente de las heces de sus mascotas y desconocen el potencial impacto en la salud de los que habitan en los hogares (Cuadro 5). En la ciudad Escárcega se han reportado parásitos de los géneros Ancylostoma y Toxocara (Encalada-Mena et al., 2011), tipo de parásitos que son considerados zoonóticos y resistentes a las condiciones ambientales (Traversa, 2012; Otero et al., 2013).

\section{Relación del Nivel Educativo con Otras Variables}

La desparasitación interna y externa demostró estar altamente relacionada con el nivel de estudio. Aunque en este trabajo no se determinó el estatus económico, existen reportes que muestran asociación entre la frecuencia de desparasitaciones y el estatus económico (Matos et al., 2015), así como una correlación positiva entre altos niveles educativos y el apego del individuo a sus mascotas (Schoenfeld et al., 2010).

Dentro de los factores asociados a las bajas cargas de parásitos en mascotas se encuentra la esterilización del animal (Visco et al., 1977; Robertson et al., 2000; Gates y Nolan, 2010), lo cual se debe a que los perros esterilizados son mejor cuidados (Balassiano et al., 2009; Smith et al., 2011). El porcentaje de animales esterilizados fue similar al reportado por Arauco et al. (2014) en Perú; sin embargo, muy bajo comparado al $60 \%$ de entrevistados reportado por Orihuela y Solano (1995) a favor de la esterilización y al 90\% reportado en países como Estados Unidos, Australia y Nueva Zelanda (Manning y Ronwan, 1992; Mackay et al., 2009; Alberthsen et al., 2016), siendo en estos países donde se aplican leyes relacionadas al bienestar de los animales de compañía. En el presente estudio, la esterilización de mascotas estuvo relacionado con el nivel educativo y podría deberse a que los entrevistados sin estudios y nivel primaria reciben apoyos gubernamentales y asisten a reuniones de programas sociales donde se les informa de apoyos como los programas de esterilización de mascotas financiados por el gobierno.

Sobre el nivel educativo y el conocimiento de zoonosis, Pereira et al. (2016) y Otranto et al. (2017) mencionan que un bajo nivel educativo se relaciona con escaso conocimiento de zoonosis. En el presente estudio, aun cuando muchos de los entrevistados tienen mayores grados de estudio, el conocimiento de las zoonosis es semejante entre los grupos evaluados. Pereira et al. (2016) señalan que las enfermedades más frecuentes que conocían sus entrevistados fueron la ehrlichiosis, sarna, leishmaniosis y las parasitosis gastroentéricas, en tanto que para Matos et al. (2015) fueron la sarna, seguida de leishmaniosis y toxoplasmosis. En el municipio de Escárcega se ha demostrado una importante prevalencia de enfermedades zoonóticas en perros con dueño (EncaladaMena et al., 2011), que incluso puede ser 
mayor en perros callejeros y que no se ha estudiado adecuadamente.

\section{Conclusiones}

- Aunque la mayoría de los dueños de mascotas administran endoparasiticidas y ectoparasiticidas a sus mascotas como una medida profiláctica contra los parásitos internos y externos, en muchos casos no se realiza en los tiempos correctos el tratamiento, haciendo necesario implementar calendarios de desparasitación precisos.

- Se deben implementar medidas de control efectivas para reducir el riesgo de infecciones parasitarias tanto en animales de compañía como en humanos.

- Se debería proporcionar más información a los propietarios de mascotas sobre las enfermedades parasitarias de las mascotas y de los riesgos de zoonosis.

\section{Literatura Citada}

1. Acosta-Jamett G, Cleaveland S, Cunningham AA. 2010. Demography of domestic dogs in rural and urban areas of the Coquimbo region of Chile and implications for disease transmission. Prev Vet Med 94: 272-281. doi: 10.1016/j.prevetmed.2010.01.002

2. Alberthsen C, Rand J, Morton J, Bennett P, Paterson M, Vankan D. 2016. Numbers and characteristics of cats admitted to Royal Society for the prevention of cruelty to animals (RSPCA) shelters in Australia and reasons for surrender. Animals 6: 23. doi: 10.3390/ani6030023

3. Alvarado-Esquivel C, Romero-Salas D, Aguilar-Domínguez M, Cruz-Romero A, Ibarra-Priego N, Pérez-deLeón $\boldsymbol{A} A$. 2015. Epidemiological assessment of intestinal parasitic infections in dogs at animal shelter in
Veracruz, Mexico. Asian Pac J Trop Biomed 5: 34-39. doi: 10.1016/S22211691(15)30167-2

4. Anuario Estadístico y Geográfico de Campeche. 2017. [Internet]. Disponible en: http://www.datatur.-sectur.gob.mx/ITxEF_Docs/CAM_ANUARIO_PDF.pdf

5. Arauco D, Urbina B, León D, Falcón N. 2014. Indicadores demográficos y estimación de la población de canes con dueño en el distrito de San Martin de Porres, Lima-Perú. Salud Tecnol Vet 2: 83-92. doi: 10.20453/stv.v2i2.2254

6. Awosanya EJ, Akande HO. 2015. Animal health care seeking behavior of pets or livestock owners and knowledge and awareness on zoonoses in a university community. Vet World 8: 841-847. doi: 10.14202/vetworld.2015.841-847

7. Balassiano BC, Campos MR, Menezes $R$ de C, Pereira MJ.2009. Factors associated with gastrointestinal parasite infection in dogs in Rio de Janeiro, Brazil. Prev Vet Med 91: 234-240. doi: 10.1016/ j.prevetmed.2009.05.030

8. Becker AC, Rohen M, Epe C, Schnieder T. 2012. Prevalence of endoparasites in stray and fostered dogs and cats in Northern Germany. Parasitol Res 111: 849-857. doi: 10.1007/s00436012-2909-7

9. Belshaw Z, Robinson NJ, Dean RS, Brennan ML. 2018. Motivators and barriers for dog and cat owners and veterinary surgeons in the United Kingdom to using preventative medicines. Prev Vet Med 154: 95-101. doi: 10.1016/j.prevetmed.2018.03.020

10. Beugnet F, Bourdeau P, ChalvetMonfray K, Cozma V, Farkas R, Guillot J, Halos L, et al. 2014. Parasites of domestic owned cats in Europe: co-infestations and risk factors. Parasite Vector 7: 291. doi: 10.1186/17563305-7-291

11. [CAPCvet] Companion Animal Parasite Council. [Internet]. Disponible en: https:/ /www.capcvet.org 
12. Chomel BB. 2014. Emerging and reemerging zoonoses of dogs and cats. Animals 4: 434-445. doi: 10.3390/ ani4030434

13. Costa-Junior LM, Rembeck K, de Melo M, Filipe L, Coelho-Azevedo S, Friche-Passos LM, Barbosa-Ribeiro MF. 2012. Occurrence of ectoparasites on dogs in rural regions of the state of Minas Gerais, Brazil. Rev Bras Parasitol V 21: 237-242. doi: 10.1186/1756-33057-291

14. Dantas-Torres F, Otranto D. 2014. Dogs, cats, parasites, and humans in Brazil: opening the black box. Parasite Vector 7: 22. doi: 10.1186/1756-3305-7-22

15. Diez M, Picavet P, Ricci R, Dequenne $M$, Renard M, Bongartz A, Farnir $F$. 2015. Health screening to identify opportunities to improve preventive medicine in cats and dogs. J Small Anim Pract 56: 463-469. doi: 10.1111/ jsap. 12365

16. Elsheikha H, Jiménez-Castro PD. 2015. Anthelmintics: targets, mechanisms and resistance. Companion Anim 20: 436-441. doi: 10.12968/coan.2015.20.8 .436

17. Encalada-Mena LA, Duarte-Ubaldo EI, Vargas-Magaña JJ, GarcíaRamírez MJ, Medina-Hernández RE. 2011. Prevalencia de parásitos gastroentéricos de cánidos en la ciudad de Escárcega, Campeche, México. Univ Cienc 27: 209-217.

18. [ESCCAP] European Scientific Counsel Companion Animal Parasites. [Internet]. Disponible en: https://www.esccap.org

19. Gates MC, Nolan TJ. 2010. Factors influencing heartworm, flea, and tick preventative use in patients presenting to a veterinary teaching hospital. Prev Vet Med 93: 193-200. doi: 10.1016/ j.prevetmed.2009.10.012

20. Halos L, Beugnet F, Cardoso L, Farkas R, Franc M, Guillot J, Pfister $K$, et al. 2014. Flea control failure? Myths and realities. Trends Parasitol 30: 228-233. doi: 10.1016/j.pt.2014.02.007
21. [INEGI] Instituto Nacional de Estadistica y Geografia. 2010. [Internet]. Disponible en: http://www.beta.-inegi.org. $\mathrm{mx} / \mathrm{app} /$ areasgeo-graficas/ ?ag=04\#tabMCcollapse

22. Kopp SR, Kotze AC, McCarthy JS, Traub RJ, Coleman GT. 2008. Pyrantel in small animal medicine: 30 years on. Vet J 178: 177-184. doi: 10.1016/j.tvj1.2007.06.021Get

23. Low VL, Prakash BK, Tan TK, SofianAzirun M, Anwar FHK, Vinnie-Siow WY, AbuBakar S. 2017. Pathogens in ectoparasites from free-ranging animals: infection with Rickettsia asembonensis in ticks, and a potentially new species of Dipylidium in fleas and lice. Vet Parasitol 245: 102-105. doi: 10.1016/j.vetpar.2017.08.015.

24. Manning AM, Ronwan AN. 1992. Companion animal demographics and sterilization status: results from a survey in four Massachusetts towns. Anthrozoös 5: 192-201. doi: 10.2752/089279392787011368

25. Matos M, Alho AM, Owen SP, Nunes T, de-Carvalho LM. 2015. Parasite control practices and public perception of parasitic diseases: a survey of dog and cat owners. Prev Vet Med 122: 174-180. doi: 10.1016/j.prevetmed.2015.09.006

26. McKay SA, Farnworth MJ, Waran NK. 2009. Current attitudes toward, and incidence of, sterilization of cats and dogs by caregivers (owners) in Auckland, New Zealand. J Appl Anim Welf Sci 12: 331-44. doi: 10.1080/10888700903163617

27. Morales MA, Varas C, Ibarra L. 2009. Caracterización demográfica de la población de perros de Viña del Mar, Chile. Arch Med Vet 41: 89-95. doi: 10.4067/S0301-732X2009000100013

28. Montañez-Escalante PI, Ruenes-Morales MR, Ferrer-Ortega MM, Estrada-Medina H. 2014. Los huertos familares Maya-Yucatecos: situación actual y perspectivas en México. Ambienta 107: 100-109. 
29. Murthy KCM, Ananda KJ, Adeppa J. 2017. Prevalence of ectoparasites in dogs of Shimoga, Karnataka. J Parasitol Dis 41: 167-170. doi: 10.1007/s12639016-0770-9

30. Orihuela TA, Solano VJ. 1995. Demographics of the owned dog population in Miacatlan, Mor. Mexico. Anthrozoös 8: 171-175. doi: 10.2752/ 089279395787156356

31. Ortega-Pacheco A, Rodriguez-Buenfil JC, Bolio-Gonzalez ME, Sauri-Arceo CH, Jiménez-Coello M, Forsberg CL. 2007. A survey of dog populations in urban and rural areas of Yucatan, Mexico. Anthrozoös 20: 261-274. doi: 10.2752/089279307X224809

32. Otero D, Nijsse R, Gomes L, Alho AM, Overgaauw P, Hoek D, Madeira-deCarvalho LM. 2014. Soil contamination with Toxocara spp eggs in public parks of Lisbon, Portugal - preliminary results. Acta Parasitol Port 20: 47-50.

33. Otranto D, Dantas-Torres F, Mihalca AD, Traub RJ, Lappin M, Baneth $G$ 2017. Zoonotic parasites of sheltered and stray dogs in the era of the global economic and political crisis. Trends Parasitol 33: 813-825. doi: 10.1016/ j.pt.2017.05.013

34. Palmer CS, Robertson ID, Traub RJ, Rees $R$, Thompson RCA. 2010. Intestinal parasites of dogs and cats in Australia: the veterinarian's perspective and pet owner awareness. Vet J 183: 358361. doi: 10.1016/j.tvj1.2008.12.007

35. Penakalapati G, Swarthout J, Delahoy MJ, McAliley L, Wodnik B, Levy K, Freeman MC. 2017. Exposure to animal feces and human health: a systematic review and proposed research priorities. Environ Sci Technol 51: 11537-11552. doi: 10.1021/acs.est.7b02811

36. Pereira A, Martins A, Brancal H, Vilhena H, Silva P, Pimenta P, DiazLopes D, et al. 2016. Parasitic zoonoses associated with dogs and cats: a survey of Portuguese pet owners' awareness and deworming practices. Parasite Vector 9: 245. doi: 10.1186/s13071-016-1533-2
37. Prates L, Otomura FH, Mota LT, Toledo MJ-de-O. 2013. Impact of antiparasitic treatment on the prevalence of ectoparasites in dogs from an indigenous territory state of Paraná, Brazil. Rev Patol Trop 42: 339-351. doi: 10.5216/rpt.v42i3.26923

38. Pullola T, Vierimaa J, Saari S, Virtala AM, Nikander S, Sukura A. 2006. Canine intestinal helminths in Finland: prevalence, risk factors and endoparasite control practices. Vet Parasitol 140: 321326. doi: 10.1016/j.vetpar.2006.04.009

39. Riggio F, Mannella $R$, Ariti G, Perrucci S. 2013. Intestinal and lung parasites in owned dogs and cats from central Italy. Vet Parasitol 193: 78-84. doi: $10.1016 /$ j.vetpar.2012.11.026

40. Rinzin K. 2007. The epidemiology of free-roaming dog and cat populations in the Wellington region of New Zealand. MSc Thesis. New Zealand: Massey University. $87 \mathrm{p}$.

41. Robertson ID, Irwin PJ, Lymbery AJ, Thompson RC. 2000. The role of companion animals in the emergence of parasitic zoonoses. Int J Parasitol 30: 1369-1377. doi.org/10.1016/j.ijppaw.2015.01.006

42. Rodríguez-Vivas RI, Cob-Galera LA, Domínguez-Alpizar JL. 2001. Frecuencia de parásitos gastrointestinales en animales domésticos diagnosticados en Yucatán, México. Rev Biomed 12: 19-25.

43. Ruiz-Izaguirre E, Eilers CHAM. 2012. Perceptions of village dogs by villagers and tourists in the coastal region of rural Oaxaca, Mexico. Anthrozoös 25: 75-91. doi: 10.2752/175303712X13240472427555

44. Sager H, Moret CS, Grimm F, Deplazes P, Doherr MG, Gottstein B. 2005. Coprological study on intestinal helminths in swiss dogs: temporal aspects of anthelminthic treatment. Parasitol Res 98: 333-338. doi: 10.1007/s00436-0050093-8

45. Schoenfeld-Tacher R, Kogan LR, Wright ML. 2010. Comparison of strength of the human-animal bond 
between Hispanic and non-Hispanic owners of pet dogs and cats. J Am Vet Med A 236: 529-534. doi: 10.2460/ javma.236.5.529

46. [SEDESOL] Secretaria de Desarrollo Social. 2013. [Internet]. Disponible en: http://www.microrregiones.gob.mx/ catloc/contenido.asp $\mathrm{x}$ ?refnac $=$ 040090001

47. Smith FD, Ballantyne R, Morgan ER, Wall R. 2011. Prevalence, distribution and risk associated with tick infestation of dogs in Great Britain. Med Vet Entomol 25:377-384. doi: 10.1111/j.13652915.2011.00954.x

48. Toribio JAL, Norris JM, White JD, Dhand NK, Hamilton SA, Malik R. 2009. Demographics and husbandry of pet cats living in Sydney, Australia: results of cross-sectional survey of pet ownership. J Feline Med Surg 11: 449461. doi: $10.1016 /$ j.jfms.2008.06.010

49. Traversa D. 2012. Pet roundworms and hookworms: a continuing need for global worming. Parasite Vector 5: 91. doi: 10.1186/1756-3305-5-91

50. Troyo A, Calderón-Arguedas Ó, Alvarado G, Vargas-Castro LE, Avendaño A. 2012. Ectoparasites of dogs in home environments on the Caribbean slope of Costa Rica. Rev Bras Parasitol 21: 179-183.

51. Visco RJ, Corwin RM,Selby LA. 1977. Effect of age and sex on the prevalence of intestinal parasitism in dogs. J Am Vet Med Assoc 170: 835-837. 